\title{
Cavernoma cerebeloso complicado con hemorragia asociado a anomalía venosa del desarrollo: reporte de caso
}

\author{
Cerebellar cavernoma complicated with hemorrhage associated with \\ developmental venous anomaly: a case report \\ Janina Franco-Chávez $\mathbb{D}^{1}$, Fiorella Chaparro-Franco $\mathbb{D}^{1}$, Alan Martínez-Chamorro $\mathbb{D}^{1}$, Oscar Ucedo $\mathbb{D}^{1}$
}

${ }^{1}$ Universidad Nacional de Asunción, Facultad de Ciencias Médicas, Santa Rosa del Aguaray, Paraguay.

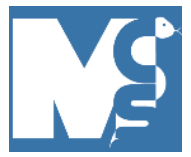

Recibido: $12 / 01 / 2021$

Revisado: 18/03/2021

Aceptado:27/04/2021

\section{Autor correspondiente}

Janina Franco-Chávez

Universidad Nacional de Asunción,

Paraguay

luzjanina98@gmail.com

\section{Conflictos de interés}

Los autores declaran no poseer conflictos de interés.

\section{Fuente de financiación}

Los autores no recibieron apoyo financiero para la investigación, autoría y/o publicación de este artículo.

Este artículo es publicado bajo una licencia de Creative Commons Reconocimiento 4.0 Internacional.

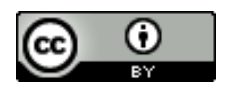

\section{RESUMEN}

El cavernoma cerebral es una lesión vascular del sistema nervioso central, la segunda más frecuente (10-15 \%) de los cuatro tipos de malformaciones cerebrales, entre las que se encuentran la malformación arteriovenosa, telangiectasia capilar y la anomalía venosa del desarrollo; asociándose a esta última en aproximadamente $9 \%$ de los casos. Su prevalencia estimada es solo del 0,4\% en la población general. Reporte de caso de mujer de 23 años de edad, que acudió al servicio de urgencias por cefalea intensa, acompañada de vértigos intensos y de vómitos en varias oportunidades; con alteración del estado de conciencia. Se realizó tomografía axial computarizada simple de cráneo, debido a la edad de la paciente y a la localización de la lesión se sospechó de una malformación vascular por lo que se procedió a realizar una resonancia magnética nuclear que demostró imágenes sugestivas de un cavernoma cerebeloso complicado con hemorragia y un angioma venoso contiguo. Se realizó tratamiento conservador y se solicitó la repetición de la RMN al mes de la primera indicación con el objetivo de valorar la evolución de la lesión y de la hemorragia en donde nada agudo ni expansivo fue visualizado. Los cavernomas frecuentemente no son detectados con una angiografía por presentar un flujo lento y por la tendencia a trombosis, siendo la herramienta diagnóstica más sensible la resonancia magnética nuclear. Se sugiere la conducta expectante frente a lesiones múltiples, a aquellas que son inaccesibles quirúrgicamente o que cursan sin complicaciones. Palabras clave: Hemangioma; Sistema Nervioso Central; Telangiectasia; Anomalías Congénitas.

\section{ABSTRACT}

The cerebral cavernoma is a vascular injury of the central nervous system, the second most frequent (10$15 \%)$ of the four types of brain malformations, among which are arteriovenous malformation, capillary telangiectasia, and developmental venous anomaly; associated with the last one in approximately $9 \%$ of cases. It is estimated prevalence is only $0.4 \%$ in the general population. Case report of a 23 -year-old woman who came to the emergency department with intense headache, accompanied by severe vertigo and vomiting in several occasions; with altered state of consciousness. A simple computerized axial tomography of the skull was performed, due to the age of the patient and the location of the injury a vascular malformation was suspected, because of which a nuclear magnetic resonance was performed, which showed images suggestive of a cerebellar cavernoma complicated with hemorrhage and a contiguous venous angioma. Conservative treatment was performed and a repeat MRI was requested a month after the first indication in order to assess the evolution of the injury and bleeding where nothing acute or expansive was visualized. Cavernomas are frequently not detected with an angiography due to their slow flow and the tendency to thrombosis, being the most sensitive diagnostic tool the nuclear magnetic resonance imaging. Watchful waiting is suggested for multiple injuries, those that are surgically inaccessible or that are uncomplicated.

Keywords: Hemangioma; Central Nervous System; Telangiectasia; Congenital anomalies. 


\section{INTRODUCCIÓN}

El angioma o cavernoma cerebral es una patología poco frecuente, pero que debe ser considerado por la presentación aguda de la sintomatología producida por sus complicaciones, que ponen de manifiesto la relevancia de su detección para realizar un tratamiento oportuno de las mismas, ya que su aparición puede ocasionar secuelas de grado variable. Se trata de una lesión vascular del sistema nervioso central, formada por colecciones de vasos sinusoidales de una sola capa que no contienen parénquima cerebral intermedio. Es la segunda más frecuente (10-15\%) de los cuatro tipos de malformaciones cerebrales, entre las que se encuentran la malformación arteriovenosa, telangiectasia capilar y la anomalía venosa del desarrollo (1); asociándose a esta última en aproximadamente $9 \%$ de los casos (2).

Esta malformación, considerada como una lesión vascular de bajo flujo (2), puede ser de aparición esporádica o familiar, de localización supratentorial (64-84\%) o infratentorial (19-35\%), teniendo una prevalencia estimada del $0,4 \%$ en la población general (1). Se presenta con síntomas variables, como cefaleas, convulsiones, déficit neurológico focales, dependiendo de la ubicación de la lesión (3). Debido a su estructura histológica, las lesiones son muy susceptibles a la trombosis y a la hemorragia (2).

A partir del avance en los estudios imagenológicos, con el uso de la resonancia magnética el diagnóstico del angioma cavernoso ha ido en aumento, por lo que la resección está indicada en los casos sintomáticos, con lesiones únicas y accesibles desde el punto de vista quirúrgico (4).

\section{PRESENTACIÓN DEL CASO}

Paciente de 23 años de edad, de sexo femenino, sin patología de base conocida, acude al servicio de urgencias por cefalea intensa, de inicio brusco en región occipital de tipo punzante que irradia a parte posterior del cuello y no cede con analgésicos comunes, acompañada de vértigos intensos que imposibilitan la estación y marcha y lateralizan a la derecha; y de vómitos en varias oportunidades de contenido alimentario, posteriormente amarillento, no precedido de náuseas; se agrega al cuadro alteración del estado de conciencia.

Al examen físico neurológico presenta un Glasgow $14 / 15$, con pupilas isocóricas, iguales, simétricas, fotorreactivas, nistagmos a derecha, resto de pares craneales conservados; adiadococinesia y dismetría negativos, sin déficit motor ni sensitivo, rigidez de nuca negativo, no se puede evaluar estación y marcha por vértigo.

Se solicita tomografía axial computarizada (TAC) simple de cráneo, llama la atención lesión hiperdensa en fosa posterior, lado derecho, que mide 1,63 x1,16 cm, con hemoventrículo que compromete al cuarto ventrículo y produce dilatación del tercer ventrículo y de astas temporales de ventrículos laterales, se observan estructuras de la línea media conservadas y surcos corticales presentes (Figura 1). En la figura se observa imagen hiperdensa en el hemisferio cerebeloso derecho, paraventricular (flecha blanca), con hemoventrículo (flecha roja) y dilatación de astas temporales de ventrículos laterales y del tercer ventrículo (flechas azules), se evidencia conservación de línea media y presencia de surcos corticales.

FIGURA 1. TAC DE CRÁNEO SIMPLE, CORTE AXIAL.

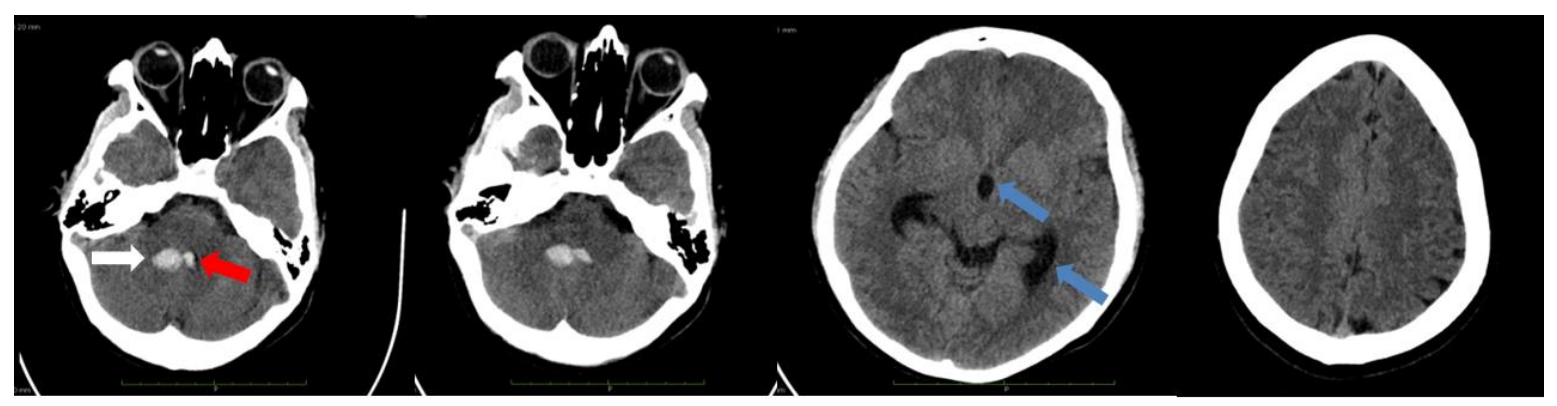

Debido a la edad de la paciente y a la localización de la lesión se sospecha de una malformación vascular por lo que se procede a realizar una resonancia magnética nuclear (RMN), que demuestra imágenes sugestivas de un cavernoma cerebeloso complicado con hemorragia y un angioma venoso contiguo: se evidencia lesión hemorrágica con hipointensidad en secuencias T2 GRE y SW1, sin edema perilesional, de $1 \mathrm{~cm}$ de diámetro; tras administración de contraste se pueden notar pequeñas estructuras vasculares realzadas, sin traducción en la secuencia TOF, indicativo de malformación de bajo flujo (Figura 2). 
FIGURA 2. RMN DE CRÁNEO CON CONTRASTE (GADOLINIO), CORTE AXIAL, CORONAL Y SAGITAL, RESPECTIVAMENTE, EN SECUENCIA T1

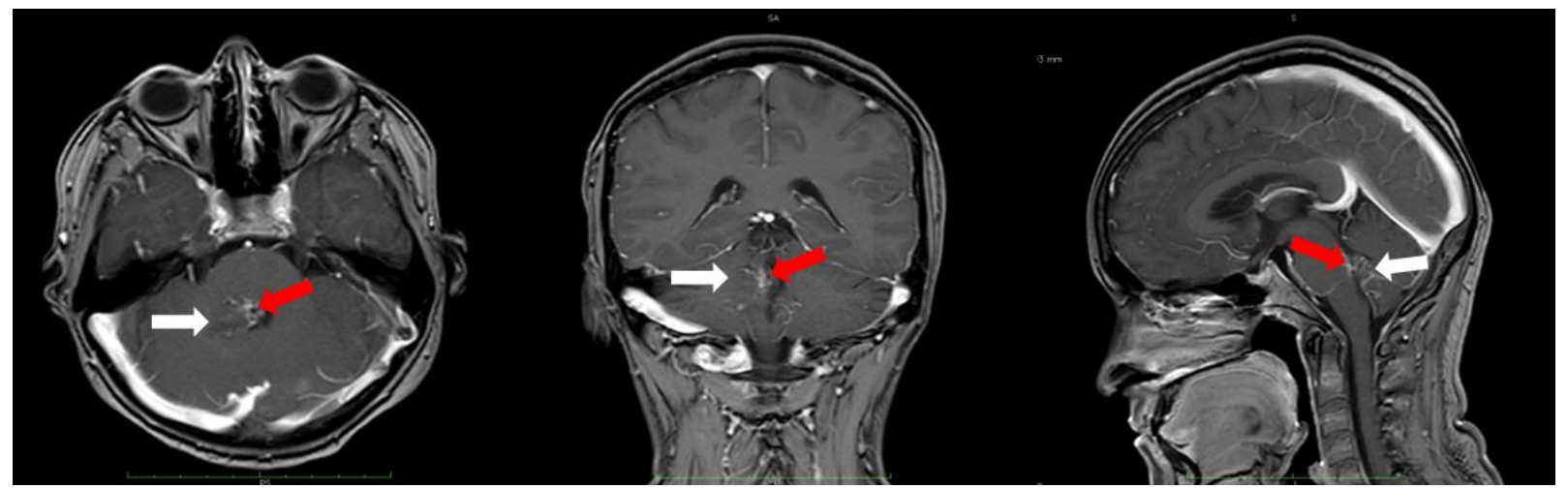

En la figura 2 se observa en la cara ventricular del pedúnculo cerebeloso medio derecho una lesión con características de cavernoma complicado con hemorragia (flechas blancas) y pequeñas estructuras vasculares regionales realzadas, contiguas, de aspecto radiado dirigidas al suelo del cuarto ventrículo (flechas rojas).

Se realiza tratamiento conservador, con medidas de cerebro agudo: cabecera elevada a 30 grados, manitol $1 \mathrm{~g} / \mathrm{kg} /$ día, manteniendo PAM entre $90-100 \mathrm{mmHg}$, con control pupilar y balance hidrosalino a horario, se repite TAC a las 24 horas evidenciando disminución de la hidrocefalia (Fig. 3), por lo que no requiere cirugía de urgencia. La paciente permanece internada durante 17 días, recibe alta médica sin secuelas. Se solicita la repetición de la RMN al mes de la primera indicación con el objetivo de valorar la evolución de la lesión y de la hemorragia, en donde nada agudo ni expansivo fue visualizado (Figura 3); actualmente se encuentra con tratamiento sintomático con betahistina ( $24 \mathrm{mg} / \mathrm{día})$, antieméticos según necesidad y en planes de exéresis del cavernoma.

FIGURA 3. A. TAC DE CRÁNEO SIMPLE, CORTE AXIAL, SE OBSERVA DISMINUCIÓN DE LA VENTRICULOMEGALIA A NIVEL DE LAS ASTAS TEMPORALES DE VENTRÍCULOS LATERALES Y DEL TERCER VENTRÍCULO. B Y C. RMN CON CONTRASTE, CORTES AXIAL Y SAGITAL, RESPECTIVAMENTE, SE EVIDENCIA LESIÓN HEMORRÁGICA SECUELAR Y SISTEMA VENTRICULAR NO DILATADO.

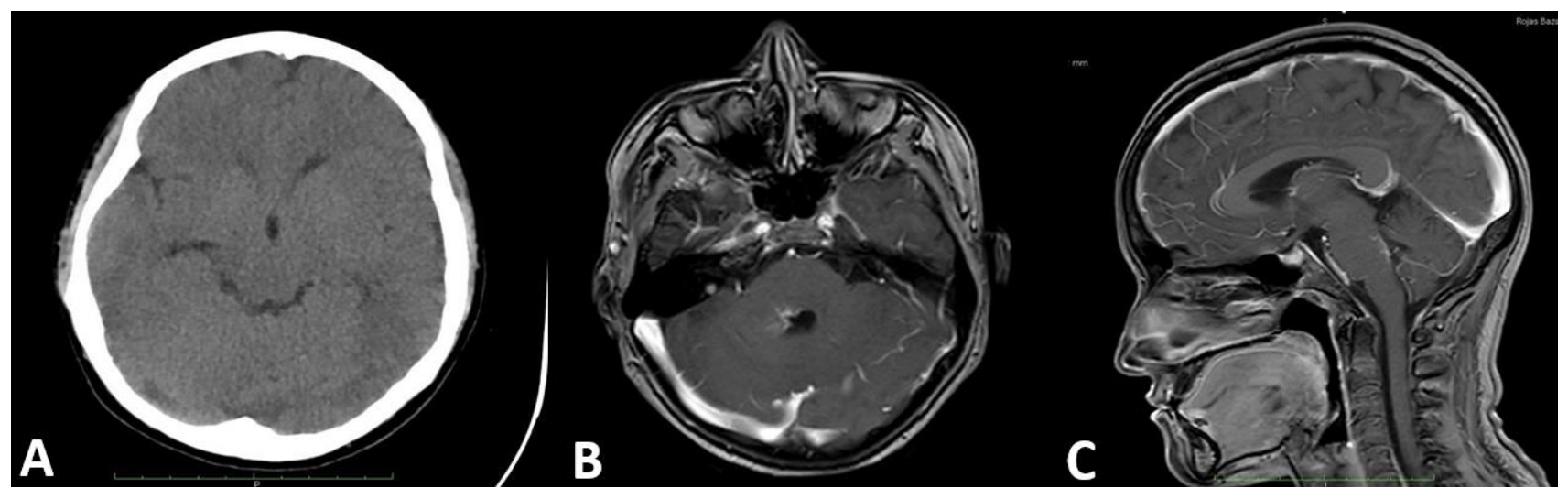

\section{DISCUSIÓN}

Los cavernomas cerebelosos son asintomáticos hasta en más del $40 \%$ de los casos, los cuales tienden a un curso más benigno en comparación a los sintomáticos (1), la sintomatología es bastante variable según la localización y el volumen de sangrado, siendo más frecuentes las crisis convulsivas, el déficit motor y la cefalea (5); las hemorragias son más comunes en aquellas lesiones asociadas a anomalías venosas del desarrollo, así como en pacientes menores de 35 años, características compatibles con el caso presentado; los pacientes más jóvenes generalmente tienen un peor pronóstico que los de edad más avanzada, asimismo, aquellos que hayan presentado hemorragia son más propensos a una segunda hemorragia (1). Según la presentación clínica, son clasificados en asintomáticos - casuales, epileptogénicos, hemorrágicos, pseudotumorales y medulares (5). 
Estas lesiones pueden ubicarse en el hemisferio cerebral (66\%), tronco encefálico (18\%), cerebelo (6 $\%)$, cuerpo calloso (1\%) o en la ínsula (0,2 \%) (2). Cuando las cerebelosas presentan sintomatología, generalmente son debidas a hemorragias en el parénquima, produciendo efecto de masa sobre estructuras adyacentes $u$ ocluyendo el cuarto ventrículo (3). Los cavernomas frecuentemente no son detectados con una angiografía por presentar un flujo lento, por lo que también son conocidos como "angiográficamente ocultos" (5), y por la tendencia a trombosis, siendo la herramienta diagnóstica más sensible la resonancia magnética nuclear. Los estudios de tomografía computarizada son utilizados para la detección de complicaciones, como la hemorragia, o para realizar el seguimiento de los pacientes con cavernoma que no han sido intervenidos quirúrgicamente (1).

Su historia natural es relativamente benigna, es posible observar hipertensión intracraneal en los que cursan con hemorragia cerebral subaguda o en los que están ubicados dentro de los ventrículos. Respecto al tratamiento, han sido descritas tres conductas generales: tratamiento clínico, quirúrgico y radioquirúrgico; se sugiere la conducta expectante frente a lesiones múltiples $o$ aquellas que son inaccesibles quirúrgicamente (5), por otro lado, la intervención quirúrgica casi nunca está indicada en las lesiones asintomáticas, considerando que el riesgo de una complicación es ampliamente superior al riesgo de una hemorragia, principalmente en aquellas lesiones que nunca han sangrado; la misma sí es recomendable en aquellas que son de fácil acceso y en las causantes de epilepsias que no son posibles de controlar con medicación anticonvulsiva (6); los pacientes jóvenes y aquellos que no tengan comorbilidades son los mejores candidatos para la cirugía (1). La radiocirugía es una opción al tratamiento de los cavernomas ubicados en áreas elocuentes con alto riesgo quirúrgico con el objetivo de reducir el riesgo de hemorragia; algunos estudios afirman que con la intervención radioquirúrgica la tasa de hemorragia anual se reduce del $32,5 \%$ al $10,8 \%$ (en los primeros dos años) y al $1 \%$ después de los dos años, asimismo, un factor a ser considerado corresponde a los efectos adversos de la radiación, los cuales se han reducido al $8 \%$ con el avance de la tecnología (7).

Se trata de una patología de escasa prevalencia, que no cuenta con un método específico de intervención; en el caso presentado no se realiza cirugía de urgencia por reducción de la hidrocefalia tras el tratamiento no intervencionista, pero es considerada la intervención quirúrgica por lo que puede afirmarse que no se aparta de las recomendaciones bibliográficas disponibles: cirugía en cavernomas cerebelosos incluso en ausencia de hemorragia (3); no se cuenta con reporte de casos similares en el país.

\section{CONTRIBUCIÓN DE AUTORES}

JFC y FCF: Aprobación de la versión final del manuscrito; diseño y planificación del estudio; redacción y edición del manuscrito; revisión crítica de la literatura; revisión crítica del manuscrito. OU: Aprobación de la versión final del manuscrito; diseño y planificación del estudio; recopilación, participación efectiva en la orientación de la investigación; participación intelectual en la conducción propedéutica y / o terapéutica de los casos estudiados.

\section{REFERENCIAS}

1. Faleiro RM, Vieira Martins LR. Cavernomas da fossa posterior do crânio - Relato de série de seis casos. Arq Bras Neurocir. 2014;33(04):352-6. https://dx.doi.org/10.1055/s-0038-1626239

2. Marnat G, Gimbert E, Berge J, Rougier M-B, Molinier S, Dousset V. Chiasmatic cavernoma haemorrhage: To treat or not to treat? Concerning a clinical case. Neurochirurgie. 2015;61(5):343-6. https://dx.doi.org/10.1016/i.neuchi.2015.05.005

3. Amato MCM, Madureira JFG, Oliveira RS de, Amato MCM, Madureira JFG, Oliveira RS de. Intracranial cavernous malformation in children: a single-centered experience with 30 consecutive cases. Arquivos de Neuro-Psiquiatria. 2013;71(4):220-8. https://dx.doi.org/10.1590/0004$\underline{282 \times 20130006}$

4. Campero Á, Baldoncini M, Villalonga J. Resección microquirúrgica de cavernoma del receso lateral derecho a través de abordaje telovelar. 2019;33:107-112. URL.

5. Marques de Almeida Holanda M, Marmo da Costa e Souza R, Kleyton Herculano de Luz S, Lisboa do Vale B, Ádrian Xavier da Silva M, Carrasco Marisca I, et al. Historia natural de 30 casos de cavernomas: un seguimiento de dos décadas en el Estado de Paraíba, Brasil. revchilneurocir. 2019;45(1):20-6. https://dx.doi.org/10.36593/rev.chil.neurocir.v45i1.5

6. Awad IA, Polster SP. Cavernous angiomas: deconstructing a neurosurgical disease. J Neurosurg. 2019;131(1):1-13. https://dx.doi.org/10.3171/2019.3.JNS181724

7. Mouchtouris N, Chalouhi N, Chitale A, Starke RM, Tjoumakaris SI, Rosenwasser RH, et al. Management of Cerebral Cavernous Malformations: From Diagnosis to Treatment. ScientificWorldJournal. 2015;2015: 808314. https://dx.doi.org/10.1155/2015/808314 